\section{LAS VÍCTIMAS DEL TERRORISMO EN ESPAÑA E IRLANDA DEL NORTE: DINÁMICAS DE SELECCIÓN DURANTE LOS "AÑOS DE PLOMO» Y POLÍTICAS DE REPARACIÓN}

\author{
María Jiménez Ramos \\ Universidad de Navarra \\ ORCID iD: https://orcid.org/0000-0002-1902-9183 \\ mjimenezr@unav.es
}

\section{VICTIMS OF TERRORISM IN SPAIN AND NORTHERN IRELAND: VICTIM SELECTION DYNAMICS DURING THE "YEARS OF LEAD» AND REPARATION POLICIES}

Cómo citar este artículo/Citation: Jiménez Ramos, M. (2019). Las víctimas del terrorismo en España e Irlanda del Norte: dinámicas de selección durante los "años de plomo» y políticas de reparación. Arbor, 195 (792): a511. https://doi.org/10.3989/ arbor.2019.792n2012

Recibido: 20 marzo 2018. Aceptado: 3 septiembre 2018.

RESUMEN: ETA y el IRA, las dos organizaciones terroristas más antiguas de Europa, llegaron a las cotas más elevadas de su actividad armada durante los "años de plomo», entre 1968 y 1981. El presente artículo pretende, por un lado, aportar una radiografía de las víctimas y de los responsables de los crímenes de este período, analizando estudios previos y revisando documentación que arroja luz sobre las dinámicas de selección de las víctimas practicadas por ambos grupos, siguiendo el modelo teórico de de la Calle y Sánchez-Cuenca. Por otro lado, ahonda en la deficiente atención institucional a los damnificados y analiza las políticas de reparación, que en ambos casos llegaron con más de tres décadas de retraso. Por último, se abordan las principales reivindicaciones pendientes, entre las que destaca el elevado nivel de impunidad de los crímenes cometidos, lo que condena a las víctimas a una victimización secundaria.

PALABRAS CLAVE: Terrorismo; víctimas del terrorismo; ETA; IRA; «años de plomo»; reparación.
Copyright: (C) 2019 CSIC. Este es un artículo de acceso abierto distribuido bajo los términos de la licencia de uso y distribución Creative Commons Reconocimiento 4.0 Internacional (CC BY 4.0).
ABSTRACT: ETA and IRA, the two oldest terrorist organizations in Europe, reached their greatest levels of violence during the "years of lead» (1968-1981). This article aims to explore the characteristics of victims in this period determining the role of the terrorist and paramilitary organisations in the generation of violence. Moreover, it tries to describe the dynamics of victim selection employed by ETA and IRA using the theoretical model of de la Calle and Sánchez-Cuenca. On the other hand, the article explores in depth the reparation policies followed by Spain and the United Kingdom, which appeared three decades after the first crimes. The victims of both countries share a common demand: to resolve the high level of impunity, which condemns victims to a secondary victimization.

KEYWORDS: Terrorism; victims of terrorism; ETA; IRA; the "years of lead»; reparation. 


\section{INTRODUCCIÓN}

El «terrorismo en países pequeños», como denomina Burleigh (2008) a los fenómenos terroristas de Irlanda del Norte y de ETA (p. 374), ha dejado consecuencias capilares en las regiones que lo han sufrido de forma continuada. Sir Kenneth Bloomfield afirmaba en el primer estudio sobre las víctimas de los conocidos metafóricamente como the troubles que la cifra de 3.600 víctimas del conflicto norirlandés podría parecer no demasiado calamitosa comparada con otros desastres del siglo XX, pero invitaba a poner lo sucedido en la escala adecuada: «El trauma de los asesinatos ha sido prolongado y ciertas comunidades lo han sufrido desproporcionadamente» (Bloomfield, 1998).

El desamparo institucional de las víctimas durante los "años de plomo», en los que se concentran los picos más elevados de asesinados, agravó los efectos de la violencia en miles de personas que aún hoy mantienen reclamaciones relacionadas con la justicia, la memoria pública o las prestaciones estatales. Este artículo pretende contribuir al conocimiento sobre la selección de las víctimas mediante la revisión de documentos y bibliografía sobre las víctimas del terrorismo en Irlanda del Norte y en España durante el mencionado periodo con el objetivo de calibrar el alcance de la violencia y de establecer las dinámicas de selección de víctimas ejercidas por los principales grupos terroristas. Para ello se utilizarán modelos de análisis teórico y documentos internos de las respectivas organizaciones. Además, el trabajo pretende analizar las políticas de reparación y ahondar en los principales asuntos pendientes.

\section{LAS VÍCTIMAS: CIFRAS GLOBALES Y DINÁMICAS DE SELECCIÓN}

ETA y el IRA tienen en común la condición de ser las dos organizaciones terroristas más antiguas de Europa, con trayectorias solo comparables en extensión a las de grupos terroristas como las FARC y el ELN colombianos o los Tigres de Liberación del Eelam Tamil en Sri Lanka ${ }^{1}$. Los primeros integrantes de ambos grupos pertenecían a una generación de jóvenes activistas que perseguían proyectos nacionalistas de carácter revolucionario y socialista y que encontraron referencias en el ámbito internacional, en concreto en los procesos de descolonización y en la lucha «antiimperialista» que estaban protagonizando algunas regiones del Tercer Mundo (Fernández Soldevilla, 2016, pp. 236-237). No obstante, existen circunstancias, como el factor étnico-religioso, la involucración del Ejército, la presencia de una amalgama de organizaciones te- rroristas de signo republicano y lealista en Irlanda del Norte o el alto nivel de vida del País Vasco, uno de los más elevados de España y de Europa, que distancian sus contextos y trayectorias.

Otro factor diferencial ha sido el número de víctimas. Aunque sin una cifra oficialmente admitida, los asesinatos cometidos por ETA alcanzan los 853 y los otros damnificados -incluidos los lesionados, amenazados, secuestrados y quienes han sufrido daños materiales en sus propiedades- se cifran en $6.389^{2}$. Del total de asesinatos, 574 se cometieron en el País Vasco. Pese a que existe cierta controversia con las cifras, el terrorismo de extrema derecha y parapolicial -este último conformado por una amalgama de siglas que estaban integradas o relacionadas con miembros de las Fuerzas y cuerpos de seguridad del Estado- asesinó a 62 personas ${ }^{3}$, lo que pone de manifiesto que ETA ha sido el principal actor responsable de la violencia terrorista en España. En el caso de Irlanda del Norte, las víctimas del IRA y de otras organizaciones republicanas rozan las 2.000 , al tiempo que se acepta el dato de que más de 3.600 personas fueron asesinadas y 30.000 resultaron heridas durante los troubles 4 .

En la mayoría de los casos las historias y las circunstancias de la muerte de las víctimas pueden ser reconstruidas. Esto no ocurre en los contextos de guerra civil, en los que se carece de información acerca de los muertos y de las motivaciones de los atacantes, debido a la magnitud del conflicto y a los intentos de los perpetradores de ocultar información que pueda incriminarlos y sirva para reparar a las víctimas. Sin embargo, la violencia terrorista permite estudiar a las víctimas de forma sistemática y a niveles micro por dos razones: las cifras de afectados, aunque elevadas, no son inabarcables a la hora de plantear un estudio detallado, y las organizaciones terroristas tienden a actuar en países desarrollados con burocracias y medios de comunicación que han dejado constancia de los actos violentos y de las personas afectadas, por lo que acceder a información relativa a los atentados, al estatus de la víctima y a las razones que la convirtieron en objetivo de los terroristas resulta plausible (Calle y Sánchez Cuenca, 2006).

También en los casos de ETA y del IRA existen sendos estudios considerados como los más exhaustivos y completos de los dedicados a las víctimas: Lost lives (McKittrtick, Kelters, Feeney y Thornton, 1999) y su homólogo en castellano, Vidas rotas (Alonso Pascual, Domínguez Iribarren y García Rey, 2010). Los dos tienen un afán enciclopédico y comparten un enfoque que combina el periodismo y la investigación histórica 
para recorrer uno a uno el devenir de todas las personas asesinadas por el terrorismo, permitiendo obtener conclusiones sobre las víctimas en el período más cruento de la actividad terrorista: los «años de plomo».

Entre 1968 y 1981 ETA -incluidas sus escisiones: ETA político-militar, berezis o comandos especiales y Comandos Autónomos Anticapitalistas- asesinó a 352 personas. La mayoría -306, el 86,9\%- murieron a manos de ETA militar, la rama de la organización que ha sobrevivido hasta su anuncio de disolución en 2018.

Entre finales de 1974 y principios de 1975 y hasta 1981 se desarrolló en España la primera etapa de la "guerra sucia» contra ETA. Más que una organización estructurada funcionó una red en la que se relacionaban miembros del Ejército, de la Policía, de la Guardia Civil y de los servicios de inteligencia, mercenarios conectados con sectores ultraderechistas, neofascistas italianos y exmilitantes de la organización terrorista francesa OAS (Organización del Ejército Secreto) (Casals, 2016, p. 120). Las acciones violentas fueron reivindicadas por varios grupos, principalmente la Triple A y el Batallón Vasco Español y las víctimas mortales ascendieron a 33. El segundo período, desde 1983 hasta finales de la década, estaría dominado por los Grupos Antiterroristas de Liberación (GAL). En total se atribuyen al terrorismo vinculado a la extrema derecha y parapolicial al menos 62 asesinatos -27 a los GAL y el resto a diferentes siglas-, lo que supondría el $7 \%$ de las víctimas relacionadas con el caso vasco (López Romo, 2015, p. 149) (véase la tabla 1).

En Irlanda del Norte, entre 1966 y 1981, el número de personas asesinadas fue de 2.398 , más del $60 \%$ del total de víctimas. La organización terrorista más letal fue el IRA provisional, a quien se atribuyen 1.160 asesinatos. Al resto de grupos republicanos -OIRA, INLA/ IPLO y otros grupos- se les responsabiliza de 214, lo que suma 1.374. Es decir, las organizaciones republicanas encabezadas por el IRA son responsables del $57 \%$ de los asesinatos cometidos en los troubles durante los «años de plomo».

Al IRA provisional le siguen dos organizaciones paramilitares de ideología lealista: el UVF (Ulster Volunteer Force) y el UDA o UFF (Ulster Defence Association o Ulster Freedom Fighters), responsables de 381 y 269 asesinatos respectivamente. Sumados a los de otros grupos lealistas, las víctimas ascienden a 703 , el $29,3 \%$ del total a lo largo del período en cuestión. En la cuantificación de las víctimas también están incluidas las causadas por el Ejército británico, que en este período suman 236, el $9,8 \%$ (véase la tabla 2).

Tabla 1. Número de asesinados por ETA y sus escisiones, así como por el terrorismo de extrema derecha y parapolicial entre 1968 y 1981

\begin{tabular}{|c|c|c|c|c|c|c|}
\hline Año & ETAm & ETApm & Berezis & CAA & ED y TP & Total \\
\hline 1968 & 2 & & & & & 2 \\
\hline 1969 & 1 & & & & & 1 \\
\hline 1970 & & & & & & 0 \\
\hline 1971 & & & & & & 0 \\
\hline 1972 & 1 & & & & & 1 \\
\hline 1973 & 6 & & & & & 6 \\
\hline 1974 & 19 & & & & & 19 \\
\hline 1975 & 12 & 4 & & & 2 & 18 \\
\hline 1976 & 16 & 1 & & & 1 & 19 \\
\hline 1977 & 8 & & 3 & & 1 & 12 \\
\hline 1978 & 63 & 1 & & 4 & 2 & 70 \\
\hline 1979 & 65 & 10 & & 5 & 5 & 85 \\
\hline 1980 & 83 & 5 & 1 & 9 & 21 & 119 \\
\hline 1981 & 30 & & & 2 & 1 & 33 \\
\hline TOTAL & 307 & 21 & 4 & 20 & 33 & 385 \\
\hline
\end{tabular}

Leyenda: ETAm (ETA militar), ETApm (ETA político-militar), Berezis (Berezis o comandos especiales), CAA (Comandos Autónomos Anticapitalistas), ED y TP (extrema derecha y terrorismo parapolicial).

Fuente: Alonso Pascual et al. (2010); López Romo (2015). 
Tabla 2. Número de víctimas mortales durante los troubles entre 1966 y 1981

\begin{tabular}{|c|c|c|c|c|c|c|c|c|c|c|c|c|}
\hline Año & IRA & OIRA & $\begin{array}{c}\text { INLA/ } \\
\text { IPLO }\end{array}$ & $\begin{array}{c}\text { Otros } \\
\text { rep. }\end{array}$ & $\begin{array}{c}\text { UDA/ } \\
\text { UFF }\end{array}$ & UVF & $\begin{array}{c}\text { Otros } \\
\text { lealistas }\end{array}$ & Ejército & $\begin{array}{c}\text { RUC/ } \\
\text { RUCR }\end{array}$ & $\begin{array}{c}\text { UDR/ } \\
\text { RIR }\end{array}$ & Otros & Total \\
\hline 1966 & & & & & & 3 & & & & & & 3 \\
\hline 1968 & & & & & & & & & & & & \\
\hline 1969 & 2 & & & 3 & & 2 & 1 & 2 & 8 & & & 18 \\
\hline 1970 & 18 & & & 3 & 1 & & & 5 & & & 1 & 28 \\
\hline 1971 & 86 & 9 & & 12 & 4 & 17 & 1 & 45 & 1 & & 5 & 180 \\
\hline 1972 & 234 & 20 & & 26 & 71 & 35 & 15 & 79 & 6 & 1 & 9 & 496 \\
\hline 1973 & 125 & 2 & & 10 & 44 & 34 & 12 & 29 & 1 & 2 & 4 & 263 \\
\hline 1974 & 129 & 7 & & 13 & 41 & 79 & 11 & 15 & 2 & & 6 & 303 \\
\hline 1975 & 94 & 9 & 12 & 15 & 20 & 100 & 1 & 6 & & & 10 & 267 \\
\hline 1976 & 139 & 1 & 5 & 18 & 50 & 71 & 6 & 14 & 2 & & 2 & 308 \\
\hline 1977 & 68 & 4 & & 2 & 12 & 14 & 2 & 7 & & & 7 & 116 \\
\hline 1978 & 60 & & & 2 & 2 & 8 & & 12 & & & 4 & 88 \\
\hline 1979 & 91 & 1 & 8 & 4 & 10 & 7 & 1 & 2 & & & 1 & 125 \\
\hline 1980 & 45 & & 8 & 5 & 9 & 4 & 1 & 7 & 2 & & 5 & 86 \\
\hline 1981 & 69 & & 11 & 4 & 5 & 7 & 2 & 13 & 3 & 1 & 2 & 117 \\
\hline TOTAL & 1160 & 53 & 44 & 117 & 269 & 381 & 53 & 236 & 25 & 4 & 56 & 2398 \\
\hline
\end{tabular}

Leyenda: OIRA (Official Irish Republican Army), INLA/IPLO (Irish National Liberation Army / Irish People Liberation Organisation), Otros rep. (otros grupos republicanos), UDA/UFF (Ulster Defence Association / Ulster Freedom Fighters), UVF (Ulster Volunteer Force), Otros lealistas (otros grupos lealistas), RUC/RUCR (Royal Ulster Constabulary / Royal Ulster Constabulary Reserve), UDR/RIR (Ulster Defence Regiment / Royal Irish Regiment), Otros (incluye fallecidos a causa de accidentes de tráfico o ataques cardíacos relacionados con los troubles). Fuente: McKittrick et al. (1999).

\subsection{Las estrategias de ETA y del IRA}

Para analizar el desarrollo de la violencia terrorista de organizaciones nacionalistas, de la Calle y Sánchez Cuenca proponen un modelo teórico con dos variables: el nivel de selección de las víctimas y la estrategia de los grupos terroristas ${ }^{5}$.

Para la selección de las víctimas, Calle y Sánchez Cuenca distinguen cinco tipos de asesinatos: los selectivos -basados en el comportamiento de la víctima, teniendo en cuenta, por ejemplo, su supuesta colaboración con las fuerzas de seguridad o su implicación en organizaciones consideradas enemigas-; los genéricos -justificados por la profesión de la víctima, como en el caso de los miembros de las fuerzas de seguridad; los indiscriminados; los colaterales -no intencionados o perpetrados habitualmente durante un atentado planificado-; y los erróneos -con frecuencia relacionados con información equivocada sobre el objetivo-.
En cuanto a la estrategia, Calle y Sánchez Cuesta definen los asesinatos de desgaste y los llevados a cabo para mantener el control. Los primeros van dirigidos contra el Estado y se materializan atacando a las fuerzas de seguridad, consideradas habitualmente «de ocupación», y a funcionarios o a personas civiles -jueces, funcionarios de prisiones, políticos, etc.-, percibidos como representantes del Estado y responsables del mantenimiento del statu quo. Los segundos se dirigen a frenar comportamientos que pueden dañar la seguridad de la organización terrorista -desertores, arrepentidos o empresarios que se niegan a caer en la extorsión- y a ganar adeptos interviniendo en problemas sociales relacionados con el tráfico de drogas a pequeña escala o con causas medioambientales. En Irlanda del Norte existirían también los crímenes sectarios debidos a motivos político-religiosos. Todos ellos estarían condicionados por los recursos disponibles y por los índices de apoyo de sus seguidores (Calle y Sánchez Cuenca, 2006). 
En lo que respecta a ETA, plasmó su estrategia en documentos internos como el Manual de formación / Formazio Eskuliburua, redactado por ETA militar en 1976 y dirigido a ETA político-militar, que había solicitado a la primera instrucciones sobre su forma de actuación. El texto resumía la dinámica de selección de víctimas practicada desde 1968 y que se mantendría durante los «años de plomo»".

ETAm argumentaba que ambas organizaciones debían cumplir una función estratégica, basada en la consecución de los objetivos políticos establecidos en la Alternativa $\mathrm{Kas}^{7}$, y otra táctica, basada en una acción militar orientada en este sentido. Para ello planteaba «una acción armada de carácter ofensivo» dirigida contra "las fuerzas e instituciones represivas». Entre ellas se incluían los "cuerpos represivos» -Guardia Civil, Policía y sus «colaboradores»- y los "poderes reales» o "poderes fácticos»-las fuerzas armadas- ${ }^{8}$. En definitiva, proponía llevar a cabo asesinatos encuadrados en la categoría de genéricos y dirigidos a amedrentar al Estado, provocando una espiral de acción-reacción-acción.

Esta dinámica parece cumplirse con el primer asesinato premeditado de ETA, el del jefe de la Brigada de Investigación Social de San Sebastián, Melitón Manzanas, el 2 de agosto de 1968. Julen Madariaga, uno de los fundadores de la organización, había amenazado a Manzanas y a otros policías en 1962 -«Pagarán caro sus crímenes. No son bravatas»- y Biltzar Ttipia (pequeña asamblea) de ETA había decidido su asesinato en una sesión el 2 de junio de 1968. Los planes de la organización se trastocaron por el asesinato no planificado del guardia civil José Antonio Pardines Arcay, quien se convertiría en su primera víctima mortal el 7 de junio de $1968^{9}$. Poco después la cúpula de ETA determinó que era el momento de atentar «contra aquel que mejor encarnaba a la represión en la mente del pueblo», en una estrategia calculada para recabar el apoyo de quienes personificaban la represión policial en la figura de Manzanas (Fernández Soldevilla, 2016, p. 166).

Tras ambos asesinatos, la fuerte represión del régimen de Franco medró sus estructuras, siguiendo el patrón de acción-reacción-acción que preveía la organización. En el transcurso de una operación policial se produjo el tercer crimen. La víctima fue el taxista Fermín Monasterio, que se negó a trasladar en su vehículo al etarra Miguel Echeverría al levantar sus sospechas por encontrarse herido. De acuerdo con la clasificación utilizada, se trató de un asesinato selectivo motivado por el hecho de que la víctima no siguió las órdenes de los terroristas. A medida que avanzan los años setenta, ETA adopta una estrategia de desgaste que eleva significativamente sus picos de violencia, siendo 1980 el año con mayor número de víctimas mortales, con un total de 98 (Alonso Pascual et al., 2010, p. 253).

Al igual que la de ETA, la estrategia del IRA respondía al patrón de guerra de desgaste contra el Estado, lo cual mantenía la lógica que movía la actividad de la organización: utilizar la violencia para forzar la expulsión de los británicos de Irlanda del Norte. En sus esquemas no entraba la destrucción del enemigo, sino causarle el mayor daño posible. El Green Book, un documento interno de obligada lectura para los potenciales militantes, afirmaba que atentar causando el mayor número de muertes y daños posibles, llevar a cabo una campaña de atentados con bomba contra intereses económicos, poner en marcha una campaña de propaganda a nivel nacional e internacional y presentar el territorio como ingobernable formaba parte de la estrategia dirigida a hacer insostenible la presencia británica en Irlanda del Norte.

Esta estrategia se desarrolla bajo condicionantes como la disponibilidad de recursos y especialmente el apoyo de la población. El modelo de de la Calle y Sánchez Cuenca plantea que la proporción de ataques indiscriminados depende del nivel de apoyo popular que reciba la organización y que cuanto mayor es dicho apoyo, más indiscriminados son los ataques. En otras palabras, la crudeza de los atentados se ajusta a los niveles de radicalización de los simpatizantes de la organización. No obstante, existe el riesgo de que los adeptos menos radicales se alejen de la línea de acción por su repulsa a ese tipo de ataques (Calle y Sánchez Cuenca, 2006).

John Stephenson, líder del IRA en 1969, explicó en sus memorias que ningún movimiento de resistencia en la historia había tenido éxito luchando por la «libertad nacional» sin algún daño colateral, pero admitía que el interés republicano por mantener el apoyo popular pasaba por causar el menor daño posible (Gambetta, 2005, pp. 219-220). Sin embargo, esto se contradice con los primeros atentados con bomba perpetrados en Belfast -dos muertos y cien heridos en un restaurante en 1972- o con el conocido como claudy bombing -nueve muertos tras la explosión de tres coches bomba-. A partir de 1974, cuando el IRA extendió sus ataques al Reino Unido en su particular venganza contra los británicos por negarse a negociar, varias bombas estallaron en dos 
pubs en Birmingham, matando a diecinueve personas. «En una zona de guerra es necesario forzar al movimiento republicano a una completa y total confianza en el apoyo de la gente», afirmó en un discurso Gerry Adams en 1977.

En Irlanda del Norte la importante presencia de grupos parapoliciales unida a los excesos cometidos por los miembros del Ejército, la existencia de un conflicto sectario que hacía patente una sed de venganza, y el contexto sociopolítico en el que los católicos han sido una minoría en un sistema dominado por los protestantes con ciertas dinámicas discriminatorias facilitaba la asimilación de atentados indiscriminados. Esta situación difiere en el caso de ETA. Su primer atentado indiscriminado fue la bomba colocada en la cafetería Rolando, en la madrileña calle del Correo, el 13 de septiembre de 1974. El objetivo eran los policías de la cercana Dirección General de Seguridad que acudían habitualmente al establecimiento. Sin embargo, solo una de las trece víctimas mortales del ataque resultó ser policía. ETA no reconoció la autoría del atentado y más tarde admitiría en documentos internos que este tipo de ataques, unidos a la posterior llegada de la transición democrática, habían «situado al pueblo en una posición más crítica contra nuestra acción». La desafección "obligaba a un tipo de acción extremadamente selectiva» que se completaba con otras «más ejemplares, ya que de lo contrario estaría en cuestión incluso la capacidad militar de ETA» (Gambetta, 2005, pp. 219-220). Por tanto, las críticas populares por los atentados indiscriminados en los que se contaban víctimas civiles habían llegado hasta la cúpula de la organización y frenado este tipo de acciones. Pese a ello, en términos de discurso público, ETA evitó cualquier referencia a la existencia de un conflicto ético surgido del uso de la violencia y construyó para sus simpatizantes un argumentario destinado a evitar críticas externas y a trasladar la responsabilidad de sus actos al enemigo (Varela Rey, Rodríguez Caballeira y Martín Peña, 2013, p. 93).

Hasta 1992 ETA mantuvo la estrategia esbozada en su Manual de formación: los cuerpos de seguridad fueron su objetivo principal junto a colectivos como funcionarios de prisiones, empresas de capital francés, representantes del poder económico español o los acusados de "txibatos» o informadores de las fuerzas de seguridad del Estado. A lo largo de su historia, el $58 \%$ de las víctimas de ETA fueron miembros de las fuerzas de seguridad y del Ejército, mientras que el $42 \%$ fueron civiles.
En Irlanda del Norte, sociedad profundamente dividida que ya había experimentado otros estallidos de ataques sectarios, los elevados niveles de violencia de finales de los sesenta y principios de los setenta fueron respondidos con políticas de seguridad, con el desplazamiento del Ejército y con el aumento de estrategias paramilitares, lo que instaló a finales de la década el conocido como «nivel aceptable de violencia» que, aunque alto y estable a lo largo del tiempo, nunca volvió a alcanzar las elevadas cotas de principios de los setenta (Smyth, 2000, p. 7). El $33 \%$ del total de víctimas de los troubles fueron civiles católicos, y el $19 \%$ civiles protestantes, lo que supone que el $52 \%$ de las víctimas fueron civiles. En los «años de plomo» el patrón se repite: el 34\% eran civiles católicos, y el $19 \%$ civiles protestantes, lo que implica que el $53 \%$ de las víctimas durante los «años de plomo» eran civiles.

En la década de los noventa ambas organizaciones cambiarían de estrategia, empujadas en buena medida por su debilitamiento y escasez de recursos, y optarían por la idea del frente nacionalista, que el IRA inició en 1994 y ETA al año siguiente. Esta nueva línea se basa en la idea de que la independencia se alcanzará mediante la presión política de todas las fuerzas nacionalistas (Calle y Sánchez Cuenca, 2004), al tiempo que se amplían los objetivos de los ataques, incluyendo a los civiles.

\section{LAS POLÍTICAS DE REPARACIÓN}

En inglés reparation tiene una doble acepción: por un lado, 'reparación' o 'desagravio'; por otro, 'indemnización'. Las políticas de reparación de las víctimas del terrorismo mantienen el significado dual del término anglosajón: tratan de reparar el daño infligido e incluyen la cuestión económica como parte necesaria en el proceso de compensación de los agravios causados por el terrorismo.

Desde la perspectiva del derecho la tutela o protección de las víctimas de delitos constituye uno de los fines específicos del proceso penal (Fernández de Casadevante Romaní, 2009). Sanz Hermida apunta a que el reconocimiento legal de la víctima debe asegurar la efectividad del derecho a la reparación, contribuir a la recuperación, aumentar la eficacia del proceso penal, y con ello el sentido individual y colectivo de la «justicia», y evitar la "victimización secundaria», definida como el "aumento innecesario del daño producido a la víctima como consecuencia del transcurso del tiempo y del propio desenvolvimiento penal», además de otras causas como la 
justificación social del acto violento o de los daños padecidos o la ausencia de respuesta institucional frente al crimen cometido (Sanz Hermida, 2009, p. 25). Desde el enfoque victimológico la «victimización secundaria» deriva de una "actitud negligente o una falta de humanidad» que provoca que la víctima se sienta «cuestionada, confundida o agobiada por los trámites, o que reviva de forma innecesaria el suceso traumático" (véase Guía general de buenas prácticas en el trato con víctimas del terrorismo que evite la victimización secundaria, 2015, p. 32). Desde el prisma psicológico otra fuente de «victimización secundaria» se encuentra en el discurso público legitimador de la violencia de los perpetradores, que con frecuencia deshumaniza al adversario y valora de forma asimétrica el sufrimiento. Estos mecanismos pueden conllevar que se presente a las víctimas como merecedoras de su padecimiento debido a sus características o a sus acciones pasadas (Martín Peña, Opotow y Rodríguez Carballeira, 2011, p. 188). Las disciplinas mencionadas coinciden en que la «victimización secundaria» agrava el daño psicológico de los damnificados y prolonga sus efectos.

Tanto en el caso de ETA como en el de las víctimas de los troubles se da esta circunstancia. Pese a que las primeras víctimas mortales aparecieron a finales de los sesenta, no fue hasta los noventa cuando los ordenamientos jurídicos de España y del Reino Unido comenzaron a tenerlas en consideración. No se trata de un fenómeno aislado. Reyes Mate habla de un «cambio epocal en la significación de las víctimas» para referirse al paso de la invisibilidad a la centralidad. Explica que el período de invisibilidad se debe a una cuestión práctica basada en que son los vivos los que tienen la posibilidad de hacer política y al convencimiento de que el progreso de la historia lleva consigo un costo humano y social. El autor considera una gran novedad histórica el movimiento que lleva a las víctimas a una posición central y asegura que el cambio se produce porque el sufrimiento deja de ser insignificante y pasa a significar injusticia (Reyes Mate, 2006). Castells menciona como clave el hecho de que ciertas sociedades europeas que habían cerrado falsamente su pasado violento comenzaran, entre las décadas de los sesenta y ochenta, a sacar a relucir las atrocidades perpetradas en torno a la II Guerra Mundial (Castells Arteche, 2017).

\subsection{La atención a las víctimas de ETA}

En España la emergencia de las víctimas se retrasó con respecto al resto de Europa hasta los años noventa, cuando el activismo de las propias víctimas y ciertos acontecimientos como el asesinato de Miguel Ángel Blanco manifestaron el ostracismo al que las instituciones públicas y la sociedad las habían relegado. Rodríguez Uribes define varias etapas en lo que a la atención institucional a las víctimas se refiere: la negación, la compasión, la solidaridad y "el tiempo de los derechos» (Rodríguez Uribes, 2013, pp. 117-190).

La negación se prolonga desde el inicio de la actividad de ETA hasta mediados de los ochenta, cuando surgen las primeras manifestaciones públicas a favor de las víctimas del terrorismo. Mientras los damnificados viven un período de irrelevancia política, moral, social y jurídica (Rodríguez Uribes, 2013, pp. 117190), ETA y su entorno ponen todos sus esfuerzos en instalar en la sociedad un discurso legitimador del terrorismo. Como contrapunto en 1981 tres mujeres fundaron la Asociación de Víctimas del Terrorismo (AVT), primera entidad que representaba los derechos de los damnificados por la violencia terrorista, con Ana María Vidal Abarca al frente ${ }^{10}$.

En el País Vasco, donde el miedo resultaba una barrera fundamental para la oposición pública al terrorismo, hubo que esperar hasta finales de la década de 1980 para que Cristina Cuesta creara la primera asociación en defensa de las víctimas, la Asociación por la Paz ${ }^{11}$. Desde entonces, y en especial desde la segunda mitad de la década de los noventa, el activismo de las víctimas ascendió de forma continuada, llegando a influir de forma determinante en las políticas públicas dedicadas al colectivo. Además, en 2002 se creó la Fundación de Víctimas del Terrorismo (http://fundacionvt.org/).

En el ámbito legislativo las primeras medidas fueron decretos de pensiones extraordinarias aprobados entre finales de los años ochenta y principios de los noventa. Destaca el real decreto ley 3/1979, de 26 de enero, de protección de la seguridad ciudadana, cuyo artículo 7 declaró a las víctimas por primera vez indemnizables de los daños sufridos a consecuencia de actos "cometidos por persona o personas integradas en grupos o bandas organizados y armados y sus conexos". Al ser la mayoría de las víctimas hasta entonces miembros de las fuerzas y cuerpos de seguridad del Estado, se les habían aplicado las normas que afectaban a los fallecidos en actos de servicio. Más tarde dos leyes fijarían el sistema indemnizatorio hasta 2011: la ley 13/1996 de medidas fiscales y del orden social, que sistematizaba y actualizaba para el futuro una primera compensación, y la 
ley 32/1999 de solidaridad con las víctimas del terrorismo, la primera que se promulgó con una mención expresa, 31 años después del primer crimen de ETA, que recogía el régimen extraordinario y de reconocimiento y aseguraba una segunda ayuda vinculada a la responsabilidad civil por el delito, estableciendo el cobro de indemnizaciones independientemente de que el condenado se declarase insolvente (Rodríguez Uribes, 2013, p. 148) ${ }^{12}$. Hasta 2016 el Estado había abonado a las víctimas del terrorismo en concepto de indemnizaciones y ayudas un total de 123.271.929 euros ${ }^{13}$.

No obstante, la norma más relevante es la ley 29/2011, de 22 de septiembre, de reconocimiento y protección integral a las víctimas del terrorismo, aplicable de forma retroactiva a todos los actos terroristas perpetrados desde el 1 de enero de 1960. La ley aspira a «un apoyo integral» que se dice «inspirado por los principios de memoria, dignidad, justicia y verdad" y confiere a la víctima un estatus normativo que comporta obligaciones vinculantes para el Estado (Muñoz Escandell, 2017, pp. 25-32). Además define de forma clara quiénes son considerados víctimas del terrorismo a efectos de derechos y prestaciones -personas fallecidas o que han sufrido daños físicos o psíquicos como consecuencia de la actividad terrorista- y reconoce el derecho a la justicia gratuita, así como medidas para consagrar el «principio de mínima lesividad en el desarrollo del proceso penal». En esta ley se establece la creación de dos órganos dedicados a la atención a las víctimas -la Oficina de Información y Asistencia a las Víctimas del Terrorismo de la Audiencia Nacional y la Dirección General de Apoyo a Víctimas del Terrorismo del Ministerio del Interior-y por primera vez se hace referencia a la memoria, estableciendo en el artículo 57 la creación de un Centro Nacional para la Memoria de las Víctimas del Terrorismo (http://www.memorialvt.com/ memorial-presentacion/), que finalmente se articuló en 2015 con la constitución de la Fundación Centro para la Memoria de las Víctimas del Terrorismo ${ }^{14}$.

Pese a que el sistema español de atención a las víctimas del terrorismo es reconocido a nivel europeo, los damnificados mantienen reclamaciones que en buena medida recoge un informe de la defensora del pueblo de 2016 (https://www.defensordelpueblo.es/wp-content/uploads/2016/12/VictimasETA. pdf $)^{15}$. Entre ellas destacan el elevado grado de impunidad -entre en el $30 \%$ y el $40 \%$ de los crímenes de ETA-, la ausencia de derecho al voto para los desterrados del País Vasco y Navarra por causa de
ETA, la diferencia indemnizatoria entre las víctimas con sentencia judicial y las que carecen de ella o la dispersión de archivos en los que se encuentra documentación relevante sobre los crímenes de ETA. Estas reclamaciones se unen a otras insatisfechas como la prohibición de los homenajes públicos a condenados por terrorismo en el País Vasco y $\mathrm{Na}$ varra, en los que se ha documentado la presencia frecuente de menores, con los consiguientes riesgos de «radicalización violenta» ${ }^{16}$.

\subsection{La atención a las víctimas de los troubles}

Las víctimas de los troubles no recibieron atención política e institucional hasta que comenzaron las conversaciones que dieron lugar al Acuerdo del Viernes Santo de 1998. Hasta entonces y desde 1964 el Reino Unido había establecido un sistema estatal de indemnización de los daños de origen delictivo -Criminal Injuries Compensation Scheme (CICS)- dedicado a compensar a víctimas de actos violentos que tuvieran lugar dentro de las fronteras del país, incluidos los actos terroristas. Su regulación legal tardó en llegar más de treinta años, cuando en 1995 se aprobó la ley de indemnización de daños penales -Criminal Injuries Compensation Act (CICA) (Muñoz Escandell, 2017, p. 57)-. En marzo de 1995 el gobierno británico había pagado un total de 1.119.585.000 libras en concepto de indemnizaciones relacionadas con los troubles, 300.516 .000 libras por daños a la propiedad y 814.219.000 libras por daños personales, en el marco de un sistema considerado insuficiente para aquellos que habían perdido seres queridos o que habían resultado heridos (Smyth, 2000, p. 15).

Con los Acuerdos del Viernes Santo, los negociadores comenzaron a hacer concesiones en torno a los presos, convertidos en un factor crucial. El gobierno de Tony Blair, previendo las críticas de las víctimas, se anticipó disculpándose y presentando el ansiado objetivo de la paz como una justificación para realizar determinadas cesiones. La liberación de los reclusos, un proceso que había comenzado en 1995, continuó y las víctimas -que, a diferencia de lo que sucedió en España, hasta entonces no se habían organizado- comenzaron a demandar servicios relacionados con la atención psicológica. Los servicios sociales elaboraron entonces el primer estudio sobre las consecuencias de los troubles en las víctimas (Department of Health and Social Services, 1998) cuyo estatus no estaba regulado. Todo ello supuso una presión creciente sobre el gobierno británico, que se vio obligado a afrontar la cuestión de los supervivientes (Smyth, 2000, p. 15). 
Se realizaron entonces dos estudios convertidos en referencia: We will remember them, dirigido por Sir Kenneth Bloomfield en 1998 por encargo de la Comisión de Víctimas de Irlanda del Norte, y The cost of the Troubles Study en 1999, un trabajo encargado a un grupo multidisciplinar que realizó una amplia labor de investigación cualitativa y cuantitativa. El estudio arrojó conclusiones relevantes acerca del alcance de lo sucedido: alrededor de 3.600 personas habían sido asesinadas, lo que implicaba que al menos 6.800 ciudadanos habían experimentado la pérdida de un familiar directo, y alrededor de 40.000 habían resultado heridas; el $91 \%$ de los muertos eran hombres y el $53 \%$ tenía menos de 29 años. El estudio adelantaba una reflexión que explicaría discrepancias posteriores en el ámbito de las víctimas: existía una «superposición» entre las categorías de "víctima» y «perpetrador», de manera que algunas víctimas se unieron a organizaciones paramilitares al menos parcialmente debido a su experiencia como damnificados (véase The costs of the Troubles Study. Final Report).

Este extremo supone, con las distancias de los contextos, una diferencia clave respecto a España, donde las víctimas nunca formaron parte de grupos parapoliciales ni utilizaron la violencia contra quienes la habían ejercido en su contra ${ }^{17}$. Por ello, en el ámbito de la legislación estatal en torno a las víctimas nunca se abrió el debate sobre la definición de 'víctima', una cuestión que en Irlanda del Norte era y sigue siendo central y motivo de agrias discrepancias: fijar esta definición implica, por un lado, establecer un determinado relato del pasado y, por otro, determinar quiénes son las personas que tienen derecho a compensaciones económicas.

La primera definición de 'víctima' la dio Bloomfield, que optó por el enfoque resumido en la frase «we are all victims», basado en que nadie que viviera en Irlanda de Norte durante los troubles pudo «escapar a algún nivel de daño». Smyth cuestiona que Bloomfield adoptara una postura en su opinión neutral que no tiene en cuenta razones empíricas, morales, políticas o prácticas que demostrarían que no todos pueden ser considerados víctimas. Afirma que las culturas contemporáneas de lealistas y republicanos están basadas en el victimismo, lo que les reporta ventajas porque la víctima per se merece simpatía, apoyo e intervención de otros para vencer al responsable de su situación. En esta lógica, cualquier ataque perpetrado por una víctima puede ser presentado como un acto de autodefensa y, por tanto, ser justificado (Smyth, 1999, pp. 27-40).
A ello se une el concepto de 'sufrimiento', también presente en la definición de Bloomfield. Smyth asegura que el sufrimiento no es una condición suficiente para otorgar la condición de víctima y pone el ejemplo de la esposa de un prisionero que sufre por la situación de su marido, pero ello no la convierte en un referente moral. La autora advierte que en sociedades profundamente divididas resulta difícil que una parte reconozca el sufrimiento de la otra y que las posturas entre los colectivos de víctimas pueden divergir. En el caso de España el sufrimiento no entra a formar parte del debate hasta que se produce un cambio de paradigma y las víctimas pasan de ser vistas como las consecuencias del conflicto a ser vistas como un colectivo que ha padecido un daño injusto. Esta nueva situación conlleva dos consecuencias: por un lado, las víctimas se convierten en figuras incómodas en tanto que sus demandas de reparación no encajan con la tendencia generalizada hacia el olvido; por otro, surge la figura del perpetrador, a quien una parte de la sociedad sigue considerando un héroe (Castells Arteche, 2017).

Discusiones aparte, la definición de 'víctima' que opera hoy en Irlanda del Norte es la que contiene la Orden de Víctimas y Supervivientes de Irlanda del Norte (Victims and Survivors Northern Ireland Order) de 2006, que establece que deben ser considerados como tales los heridos física o psicológicamente, aquellos que proporcionan cuidados a los anteriores debido a un acto violento y aquellos que han perdido a un familiar directo a consecuencia de algún acto violento. Esta definición ha permanecido inamovible desde 2006, pese a las voces críticas que argumentan que, dada la amplitud de la redacción, en la práctica supone una igualación entre víctimas y verdugos. En la actualidad, la estructura de apoyo a las víctimas se sustenta en un sistema tripartito formado por el Servicio de Víctimas y Supervivientes (Victims and Survivors Service), la Comisión para las Víctimas y Supervivientes de Irlanda del Norte (Commission for Victims and Survivors Northern Ireland, CVSNI) y el Forum de Víctimas y Supervivientes (Victims and Survivors Forum). El trabajo de los dos primeros está supervisado por la Unidad de Víctimas y Supervivientes (Victims and Survivors Unit), perteneciente al gobierno de Irlanda del Norte. El Victims and Survivors Service se encarga de la asistencia a las víctimas y de dar financiación a otros grupos que atienden necesidades específicas. El movimiento asociativo creció tarde y rápido, de manera que el $75 \%$ de las asociaciones se fundaron después del alto el fuego de 1994. Su división en 
asociaciones nacionalistas, unionistas o transversales es un reflejo de la dinámica vigente durante los troubles (Argomaniz, 2017).

Otras de las reclamaciones de las víctimas, al igual que en el caso vasco, tienen que ver con la impunidad. El Acuerdo del Viernes Santo establecía que si alguien era hallado culpable de un asesinato cometido antes de 1998, podía ser condenado y enviado a prisión por un período máximo de dos años. Durante el segundo de esos dos años podía ser liberado para reintegrarse en la sociedad. Además Funston denuncia que el Estado se niega a reabrir investigaciones sobre crímenes y que los responsables, ya sean republicanos o lealistas, se niegan a colaborar con las víctimas para que puedan saber la verdad, negándoles el derecho a la justicia y a la verdad ${ }^{18}$. Se trata de un nuevo ejemplo de victimización secundaria.

\section{CONCLUSIONES}

Pese al elevado nivel de incidencia que el terrorismo ha tenido en regiones pequeñas como el País Vasco e Irlanda del Norte, los niveles de violencia alcanzados en ambas no son comparables. Mientras que entre 1968 y 1981 ETA asesinó a 352 personas -la mayoría en el País Vasco-, los asesinatos perpetrados en el contexto de los troubles son 2.358, teniendo además en cuenta que el Ulster tiene una población que equivale aproximadamente a dos tercios de la del País Vasco. La presencia de grupos parapoliciales que actuaron contra ETA es reducida en términos generales, al contrario de lo ocurrido en Irlanda del Norte, donde hubo una fuerte presencia de estos grupos y donde además entraba en juego un factor diferencial ausente en el País Vasco: el conflicto étnico-religioso.

El modelo teórico de dinámicas de selección de víctimas establecido por Calle y Sánchez Cuenca, apoyado por la consulta de manuales internos de ETA y del IRA, explica que en ambos casos las organizaciones desarrollaran una estrategia de desgaste contra el Estado, provocando una espiral acción-reacciónacción que aumentó sus niveles de apoyo popular. El cambio de estrategia solo se produciría a mediados de los años noventa, cuando ambas organizaciones optarían por la idea del frente nacionalista, aunando la presión política de todas las fuerzas nacionalistas con la ampliación de sus objetivos a civiles. Hasta entonces, mientras los documentos internos de ETA Ilamaban a perpetrar atentados selectivos principal- mente contra miembros de las fuerzas de seguridad ante el temor de que el asesinato de civiles redujera su apoyo ciudadano, los líderes del IRA invitaban a hacer el mayor daño posible, lo que en la práctica se traducía en niveles más elevados de atentados indiscriminados. El factor que explica esta diferencia es la etnicidad presente en una población dividida por la religión, lo que contribuye a la radicalización de los simpatizantes. De acuerdo con la ecuación de Calle y Sánchez Cuenca, cuanto más elevado es el nivel de radicalización, mayor es la tolerancia con las acciones indiscriminadas, incluso si hay población civil entre las víctimas, aunque este tipo de ataques puede conllevar un coste en términos de apoyo de los sectores menos radicales.

La atención a las víctimas tanto en España como en Irlanda del Norte fue deficiente durante los años de mayor actividad terrorista. No existían normas específicas y las compensaciones que recibieron se basaron en normas establecidas para otras circunstancias. Las medidas diferenciadas llegaron con treinta años de retraso. Mientras que el sistema de atención en España es uno de los más desarrollados de Europa, en Irlanda del Norte existe una cuestión fundamental que todavía hoy es objeto de polémica: la definición de 'víctima', cuyo establecimiento implica la fijación de una narrativa de los troubles.

Un asunto pendiente en ambos contextos es la impunidad que, además de un déficit en el sistema judicial, constituye un factor para la victimización secundaria. En España se calcula que alrededor del $40 \%$ de los asesinatos de ETA están sin resolver (Fernández Calderín, 2015). En Irlanda del Norte, con índices de impunidad mucho más elevados, la gravedad de este extremo se acentúa debido a la importante rebaja de penas acordada en los Acuerdos del Viernes Santo y al escaso interés del Estado por aclarar los crímenes ${ }^{19}$, lo que ha infundido entre las víctimas la sensación de una impunidad irrevocable.

\section{AGRADECIMIENTOS}

Este trabajo se enmarca dentro de los proyectos de investigación financiados por el Ministerio de Economía y Competitividad El terrorismo europeo en los años de plomo: un análisis comparativo, HAR201565048-P, cuyo investigador principal es Juan Avilés, y Perfiles del centro político (1976-1986), HAR201675600-C2-1-P, cuyo investigador principal es Pablo Pérez López. 


\section{NOTAS}

1. Véase la base de datos $A$ world of terror Exploring the reach, frequency and impact of terrorism around the world. Disponible en http://terror.periscopic.com.

2. Datos del Ministerio del Interior a fecha de junio de 2017. Véase http:// www.europapress.es/nacional/noticiacenso-interior-reconoce-10181-victimas-terrorismo-cifra-853-asesinadoseta-20170618102154.html

3. Dato extraído del Informe Foronda (López Romo, 2015, p. 164) que incluye a las víctimas mortales causadas por los Grupos Antiterroristas de Liberación (27), Batallón Vasco Español (16), Grupos Armados Españoles (6), Triple A (8) y otras organizaciones de extrema derecha (5).

4. Datos extraídos de Bloomfield (1998) y de Sánchez Cuenca y Calle Robles (2010).

5. Este trabajo, a su vez, se apoya en la base de datos The Domestic Terrorist Victims dataset (Sánchez Cuenca y Calle Robles, 2010) y en The victims of ETA dataset, disponible en https://ic3jm.es/investigacion/proyectos/explaining-terrorist-and-insurgent-behavior/\#domestic.

6. Informe n. 53/2014 de la Jefatura de Información de la Unidad Central Especial (U.C.E.) 1 de la Guardia Civil.

7. El manifiesto Alternativa Kas, publicado en 1976, recogía los objetivos estratégicos que ETA ha mantenido a lo largo de su trayectoria: retirada de Euskadi de la Policía Nacional y de la Guardia Civil; adopción de medidas para mejorar las condiciones de los trabajadores; reconocimiento de la soberanía nacional de Euskadi y del euskera como idioma ofi- cial; sometimiento del gobierno vasco a las fuerzas de defensa ciudadanas que sustituyan a las del Estado; y poderes para dotarse de sus propias estructuras económicas, sociales y políticas.

8. Informe n. 0 53/2014 de la Jefatura de Información de la Unidad Central Especial (U.C.E.) 1 de la Guardia Civil.

9. Sobre el asesinato de José Antonio Pardines, véase Fernández Soldevilla (2016, 7 de junio)

10. Comunicación personal en entrevista a Ana María Vidal Abarca realizada en Madrid el 30 de enero de 2015.

11. Comunicación personal en entrevista a Cristina Cuesta realizada en Madrid el 3 de junio de 2016.

12. Acerca de este asunto, el Colectivo de Víctimas del Terrorismo (COVITE) demandó en mayo de 2016 un protocolo que hiciera efectivo el pago de las indemnizaciones por terrorismo por parte de los condenados, que el Estado adelantaba sin que después los condenados las abonaran al declararse insolventes. Véase https://covite.org/ destacada/covite-insta-al-estado-a-asegurar-que-condenados-por-terrorismopaguen-las-indemnizaciones/.

13. Véase Anuario Estadístico del Ministerio del Interior 2016. [En línea]. Disponible en http://www.interior.gob.es/web/ archivos-y-documentacion/anuarioestadistico-de-2016.

14. En el ámbito regional cabe mencionar Gogora, Instituto para la Memoria, la Convivencia y los Derechos Humanos, impulsado por el Gobierno vasco, cuyo planteamiento incluye el reconocimiento de las víctimas de la guerra civil, lo que ha suscitado críticas entre algunos sectores. Véase Segovia (2017, 19 de febrero).

15. Sobre los crímenes sin resolver puede consultarse también el Informe actualizado de la Fiscalía de la Audiencia Nacional sobre causas penales con víctimas del terrorismo de 13 de diciembre de 2011.

16. COVITE ha presentado ante Naciones Unidas dos informes en los que aborda este asunto: Informe sobre los efectos del terrorismo en el disfrute de los Derechos Humanos, de septiembre de 2016, y Terrorismo y procesos de radicalización violenta en niños y niñas: dos importantes barreras para lograr el desarrollo sostenible, de 14 de octubre de 2016.

17. Resulta conveniente apuntar que se han dado algunos casos de victimarios pertenecientes a ETA que se convirtieron en víctimas del terrorismo parapolicial o de extrema derecha. La aplicación del Convenio Europeo de 1983 prevé reducir o suprimir la indemnización si la víctima o el solicitante participan en la delincuencia organizada o pertenecen a una organización que se dedica a perpetrar delitos violentos.

18. Conferencia de Ken Funston en el seminario Reclaiming the past, shaping the future, organizado por la South East Fermanagh Foundation (SEFF) en Fermanagh el 4 de marzo de 2017.

19. En 2015 el secretario para Irlanda del Norte, Peter Hain, declaró que los crímenes sin resolver debían «mantenerse irresueltos» y que una amnistía de facto era necesaria para dejar atrás el pasado (Rawlinson, 2014, 7 de abril).

\section{BIBLIOGRAFÍA}

Alonso Pascual, R., Domínguez Iribarren, F. y García Rey, M. (2010). Vidas rotas. Historia de los hombres, mujeres y niños víctimas de ETA. Madrid: Espasa.

Argomaniz, J. (2017). The impact of ethno-nationalist violence: Comparing the experiences of victims of ETA and paramilitaries in Northern Ireland. En Leonisio, R., Molina, F. y Muro, D. (eds.) ETA's Terrorist Campaign. From Violence to Politics, 1968-2015. London: Routledge, pp. 125-142. https://doi. org/10.4324/9781315657806-9
Bloomfield, K. (1998). We will remember them. Report of the Northern Ireland Victims Commissioner. [En línea]. Disponible en https://cain.ulster.ac.uk/ issues/victims/docs/bloomfield98.pdf

Burleigh, M. (2008). Sangre y rabia. Una historia cultural del terrorismo. Madrid: Taurus.

Calle, L. de la y Sánchez Cuenca, I. (2004). La selección de víctimas en ETA. Revista Española de Ciencia Política, 10, pp. 53-79.
Calle, L. de la y Sánchez Cuenca, I. (2006). The production of terrorist violence: analyzing target selection within the IRA and ETA. Estudios / Working Papers (Centro de Estudios Avanzados en Ciencias Sociales), 230

Casals, X. (2016). La Transición española. El voto ignorado de las armas. Barcelona: Pasado y Presente.

Castells Arteche, L. (2017). Las víctimas en la historia. En Mateo Santamaría, E. y Rivera Blanco, A. (eds.) Víctimas. ¿Todas 
iguales o todas diferentes? Caracterización y respuestas ante un fenómeno complejo. Vitoria: Fundación Fernando Buesa Blanco e Instituto Universitario Valentín de Foronda, pp. 65-88.

Department of Health and Social Services (1998). Living with the trauma of the troubles: report on a developmental project to examine and promote the further development of services to meet the social and psychological needs of individuals affected by civil unrest in Northern Ireland. Belfast: Department of Health and Social Services of Northern Ireland.

Fernández Calderín, J. F. (2015). Agujeros del sistema. Más de 300 asesinatos de ETA sin resolver. Vitoria: Iskusager.

Fernández de Casadevante Romaní, C. (2009). Las víctimas y el Derecho internacional. Anuario de Derecho Internacional, XXV, pp. 3-66.

Fernández Soldevilla, G. (2016). La voluntad del gudari. Génesis y metástasis de la violencia de ETA. Madrid: Tecnos.

Fernández Soldevilla, G. (2016, 7 junio). José Antonio Pardines, la primera víctima mortal de ETA. [En línea]. Disponible en https://observatorioterrorismo. com/historia/jose-antonio-pardines-laprimera-victima-mortal-de-eta/

Gambetta, D. (2005). Making sense of suicide missions. Oxford: Oxford University Press. https://doi.org/10.1093/acprof:o so/9780199276998.001.0001

López Romo, R. (2015). Informe Foronda. Los contextos históricos del terrorismo en el País Vasco y la consideración social de sus víctimas. 1968-2010. Madrid: Los Libros de la Catarata.

Martín Peña, J., Opotow, S. y Rodríguez Carballeira, A. (2011). Amenazados y víctimas del entramado de ETA en Euskadi: un estudio desde la teoría de la exclusión moral. Revista de Psicología Social, 26 (2), pp. 177-190. https://doi. org/10.1174/021347411795448992

McKittrick, D., Kelters, S., Feeney, B. y Thornton, C. (1999). Lost lives. The stories of the men, women and children who died as a result of the Northern Ireland troubles. Edinburgh: Mainstream.

Muñoz Escandell, I. (2017). Estatuto jurídico de las víctimas del terrorismo en Europa: Estudio de Derecho Comparado. [En línea]. Disponible en https:// iugm.es/wp-content/uploads/2017/06/ Estatuto-juri\%CC\%81dico-de-lasvi\%CC\%81ctimas.pdf?id=3145

Rawlinson, K. (2014, 7 de abril). Troubles crimes should be 'left unsolved', says former NI secretary. The Guardian. [En línea] Disponible en: https://www.theguardian.com/uk-news/2014/apr/07/ troubles-crimes-left-unsolved-peterhain

Reyes Mate, M. (2006). Justicia de las víctimas y reconciliación en el País Vasco. [En línea]. Disponible en https://www. fundacionalternativas.org/public/storage/laboratorio_documentos_archivos/ xmlimport-TTg1rD.pdf

Rodríguez Uribes, J. M. (2013). Las víctimas del terrorismo en España. Madrid: Dykinson.

Sánchez Cuenca, I. y Calle Robles, L. de la (2010). The Domestic Terrorist Victims dataset. [En línea]. Disponible en https://dataverse.harvard.edu/dataset. xhtml?persistentld=doi:10.7910/DVN/ HJHGYA

Sanz Hermida, A. M. (2009). Víctimas de delitos: derechos, protección y asistencia. Madrid: lustel.

Segovia, M. (2017, 19 de febrero). Euskadi y Madrid, entre dos memorias. El Independiente. [En línea]. Disponible en: https://www.elindependiente.com/ politica/2017/02/19/euskadi-y-madridentre-dos-memorias/

Smyth, M. (1999). Remembering in Northern Ireland: Victims, Perpetrators and Hierarchies of Pain and Responsibility. In: Hamber, B. (ed.). Past Imperfect: Dealing with the Past in Northern Ireland and Societies in Transition. Derry Londonderry: Incore.
Smyth, M. (2000). Lost lives: victims and the construction of 'victimhood' in Northern Ireland. In Cox, M., Guelke, A. y Stephen, F. (eds.). A farewell to arms? Beyond the Good Friday Agreement. Manchester: Manchester University Press.

The costs of the Troubles Study. Final report. The cost of the Troubles Study. April 1999. [En línea]. Disponible en: http://conflictresearch.org.uk/reports/ legacy-of-the-conflict/COST-OF-THETROUBLES-STUDY.pdf

Varela Rey, A., Rodríguez Caballeira, A. y Martín Peña, J. (2013). Psychological analysis of ETA's violence legitimation discourse. Revista de Psicología Social, 28 (1), pp. 85-97. https://doi. org/10.1174/021347413804756050

\section{Otros recursos}

Colectivo de Víctimas del Terrorismo (COVITE). Informe sobre los efectos del terrorismo en el disfrute de los Derechos humanos. [En línea]. Disponible en https://covite.org/wp-content/ uploads/2016/09/COVITE_Informeefectos-del-terrorismo-en-DDHH.pdf

Guía general de buenas prácticas en el trato con víctimas del terrorismo que evite la victimización secundaria. Junio de 2015. [En línea]. Disponible en http:// www.euskadi.eus/contenidos/proyecto/ victimas_proyecto006/es_def/adjuntos/ Guia_general_buenas_practicas.pdf

Irish Republican Army Green Book. [En línea]. Disponible en: http://cain.ulst.ac.uk/ othelem/organ/ira/ira_green_book.htm

Terrorismo y procesos de radicalización violenta en niños y niñas: dos importantes barreras para lograr el desarrollo sostenible. Aportaciones de COVITE para el Informe de la Oficina del Alto Comisionado de la ONU para los Derechos $\mathrm{Hu}$ manos (ACNUDH) sobre la protección de los derechos del niño en la implementación de la Agenda 2030 para el Desarrollo Sostenible. [En línea]. Disponible en https://www.ohchr.org/Documents/ Issues/Children/2030/COVITE.pdf 